\title{
Understanding suicide risk and eating disorders in college student populations: Results from a National Study
}

\author{
Sarah K. Lipson ${ }^{1}$ | Kendrin R. Sonneville ${ }^{2}$
}

${ }^{1}$ Department of Health Law Policy and Management, Boston University School of Public Health, Boston, Massachusetts

${ }^{2}$ Department of Nutritional Sciences, University of Michigan School of Public Health, Ann Arbor, Michigan

\section{Correspondence}

Sarah K. Lipson, Department of Health Law Policy and Management, Boston University School of Public Health, Boston, MA 02118. Email: skilpson@bu.edu

\begin{abstract}
Objective: To examine suicide risk by eating disorder severity and symptom presentation in a nationwide sample of college students.

Method: The Healthy Minds Study is the largest mental health survey of college populations in the United States. We analyzed the most recent available data (2015-2017) with 71,712 randomly selected students from 77 campuses. We estimated associations between two measures of suicidality (ideation and attempts) and three validated measures of eating disorder symptoms (the SCOFF, weight concerns scale, and the eating disorder examination questionnaire binge and purge items). Importantly, we also controlled for co-occurring symptoms of depression and anxiety, based on validated screening tools. The large, diverse sample provided a unique opportunity to assess whether certain individual characteristics were associated with increased risk.

Results: Eating disorder symptoms, even at subthreshold levels, were highly predictive of suicidality. Relative to students with no apparent eating disorder symptoms, students with the highest symptom levels (a SCOFF score of 5) had 11 times higher odds of attempting suicide, while those with subthreshold symptoms had two times higher odds. We also observed a strong association between suicide attempts and eating disorder presentations that included purging. Students from marginalized backgrounds, particularly gender and sexual minorities, were at increased risk for suicide and eating disorders.

Discussion: In the largest known study to date, findings suggest that eating disorders should be a priority within broader campus suicide prevention efforts, should be assessed along a continuum of severity and symptom presentation, and should focus on reaching vulnerable students.
\end{abstract}

\section{KEYWORDS}

college students, eating disorders, national survey, psychiatric comorbidity, suicidal ideation, suicide attempted

\section{1 | INTRODUCTION}

Eating disorders have the highest rate of mortality of any mental illness, and suicide risk is substantially elevated among individuals with eating disorders compared with the general population (Fichter \&
Quadflieg, 2016; Herzog et al., 2000; Pisetsky, Thornton, Lichtenstein, Pedersen, \& Bulik, 2013; Suokas et al., 2013). Suicide accounts for an estimated one-in-five deaths among individuals with anorexia nervosa, making it the second leading cause of mortality (Arcelus, Mitchell, Wales, \& Nielsen, 2011; Sullivan, 1995). Numerous studies 
document high rates of attempted suicide in patients with anorexia and bulimia nervosa as well as binge eating disorder (Conti et al., 2017; Favaro \& Santonastaso, 1997; Forrest, Zuromski, Dodd, \& Smith, 2017; Franko \& Keel, 2006; Wade, Fairweather-Schmidt, Zhu, \& Martin, 2015; Yao et al., 2016). Suicide risk is further elevated among individuals with comorbid psychiatric conditions, such as depression and anxiety (Bodell, Joiner, \& Keel, 2013; Franko \& Keel, 2006; Yao et al., 2016).

While the relationship between eating disorders and suicidality is well documented in clinical samples (Arcelus et al., 2011; Conti et al., 2017; Favaro \& Santonastaso, 1997; Forrest et al., 2017; Franko \& Keel, 2006; Sullivan, 1995; Wade et al., 2015; Yao et al., 2016), considerably less is known about this relationship at a population level. Eating disorders are undertreated and underdiagnosed (Udo \& Grilo, 2018), meaning that studies in clinical settings capture only a portion of the true burden of disease. Population-level data on eating pathology and suicide risk are sorely lacking, creating barriers to advancement in intervention and prevention efforts. Furthermore, like all mental health conditions, eating disorders exist on a continuum of symptom severity from clinically severe to subthreshold. A small but consistent body of research shows a strong correlation between suicide risk and subthreshold eating disorder symptoms (or "disordered eating"), such as overeating (Ackard, Neumark-Sztainer, Story, \& Perry, 2003), dietary restriction (Rafiroiu, Sargent, Parra-Medina, Drane, \& Valois, 2003), weight control behaviors (Crow, Eisenberg, Story, \& Neumark-Sztainer, 2008a; Crow, Eisenberg, Story, \& Neumark-Sztainer, 2008b), and body dissatisfaction (Crow et al., 2008a; Rodríguez-Cano, Beato-Fernández, \& Llario, 2006). From a public health perspective, research is needed to examine suicide risk across the continuum of eating disorder severity and symptom presentation at a population level. Such research is particularly important during the epidemiologically vulnerable adolescent and young adult years.

College students are an important population for prevention and early intervention of both eating disorders and suicide. The traditional college years (ages 18-25) directly coincide with the median age of onset for eating disorders (Franko \& Keel, 2006), and more than 1,100 students die by suicide each year (Fernández Rodríguez \& Huertas, 2013), making suicide the second leading cause of death in college populations nationwide (Schwartz, 2006). Public health research on college mental health has focused primarily on the prevalence and cooccurrence of suicide, depression, anxiety, and substance use. While important studies have examined the prevalence of eating disorders in specifically defined student subgroups (e.g., sorority women, female athletes, students from certain academic departments such as nutrition, psychology, and dance; Allison \& Park, 2004; Harris, Gee, D'Acquisto, Ogan, \& Pritchett, 2015; Wollenberg, Shriver, \& Gates, 2015), these usually rely on nonrandom samples (Eichen et al., 2016; Goel et al., 2018) or are single-site studies with limited generalizability. Considering the full body of evidence on college student mental health, significantly less is known about eating disorders relative to other mental health problems common in college populations despite the importance of this time period for prevention and intervention efforts.
Large-scale studies with representative samples are needed in order to understand relationships between suicide risk and eating disorder symptoms in college student populations. Such knowledge is essential for understanding who is most at risk, the specific clustering of symptoms most strongly associated with suicidality, and how different pathologies present in combination with suicidal ideation and suicide attempts. This study draws from a randomly selected, national sample of college students on over 75 campuses across the United States and uses validated measures to assess eating disorders and suicide risk. This is the largest known study to date and contributes new evidence to advance understanding and inform prevention and intervention efforts.

\section{2 | METHOD}

\section{1 | Data}

The Healthy Minds Study (HMS) is an annual, web-based survey focused on mental health and related factors in undergraduate and graduate student populations (Eisenberg \& Lipson, 2019). We analyzed the most recently available years of data, collected from fall 2015 through spring 2017. Colleges and universities elected to enroll in HMS; there were no exclusion criteria for institutional enrollment. At each institution with $\geq 4,000$ students, the HMS team recruited a random sample of $4,000^{\circ}$-seeking students from the full population; at smaller institutions, all students were recruited. Recruitment was conducted by email. Students had to be at least 18 years old to participate; there were no other exclusion criteria. To incentivize participation, students were informed of their eligibility for one of several prizes totaling $\$ 2,000$ annually (ten $\$ 100$ and two $\$ 500$ gift cards per year). Upon clicking a personalized link in the email, students were presented with an informed consent page and had to agree to the terms of participation before entering the survey. HMS data were collected over a month-long period at each campus using the Qualtrics software, and the survey took students approximately $15 \mathrm{~min}$ to complete.

Healthy Minds Study was administered as a series of modules (survey sections) focused on specific topics. At each institution, the survey was comprised of five total modules: three standard modules administered on all participating campuses (demographics, mental health status, and mental health service utilization) plus two elective modules chosen by the school from a menu of 13 options, including one eating disorder elective module. As described below, we took advantage of data from both the standard and elective modules to present the most comprehensive evidence to date around eating disorders and suicide risk. We analyzed data from the standard modules (henceforth, Study A) as well as data collected at campuses that administered the eating disorder module (Study B). The eating disorder measures examined in Study B (see Section 2.3) were not part of the standard modules and were only assessed for students on campuses that chose this elective. Thus, the analytic sample for Study B is a subset of the larger sample from Study A. Students in Study B were asked the same demographic questions as those in Study A. 
All research was approved by the Institutional Review Boards at participating institutions. To further protect respondent privacy, the study was covered by a Certificate of Confidentiality from the National Institutes of Health.

\section{2 | Nonresponse analysis}

Healthy Minds Study response rates were $27 \%$ in academic year 2015-2016 and 23\% in 2016-2017. Though this is typical of online surveys in this population (Eisenberg, Golberstein, \& Gollust, 2007), a potential concern is that respondents were not fully representative of the population from which they were drawn. To adjust for potential differences between respondents and nonrespondents, we constructed sample probability weights. For all students in the initial random samples, the HMS team obtained administrative data from Registrars at participating institutions, including gender, academic level, race/ethnicity, and grade point average. We used these data to construct response weights, equal to 1 divided by the predicted probability of survey response, with a logistic regression estimating probability of response based on these variables. The following characteristics were associated with response propensity: female, higher GPA, and graduate student status. Thus, weights were larger for respondents with underrepresented characteristics, ensuring that all estimates are representative of the full population in terms of basic demographic and other characteristics.

\section{$2.3 \mid$ Measures}

\subsection{1 | Eating disorder symptoms}

In Study A, the eating disorder measure is the five-item SCOFF, a widely used screening tool (Morgan, Reid, \& Lacey, 1999). The items are as follows: (a) "Do you make yourself sick because you feel uncomfortably full?," (b) "Do you worry that you have lost control over how much you eat?," (c) "Have you recently lost more than 15 pounds in a three-month period?," (d) "Do you believe yourself to be fat when others say you are too thin?," and (e) "Would you say that food dominates your life?". Response options are "yes" (1) or "no" (0). Scores range from 0 to 5 , with $\geq 2$ considered a positive screen. Prior studies have determined this cut-off to be both sensitive (72-100\%) and specific (73-94\%) for the diagnosis of anorexia and bulimia nervosa (Luck et al., 2002; Morgan et al., 1999). Recent research found the SCOFF to have $70 \%$ sensitivity and $78 \%$ specificity for binge eating disorder (Maguen et al., 2018).

In Study B, symptoms were based on two validated measures: (a) the weight concerns scale (WCS; Killen et al., 1994), and (b) the binge eating and purging items from the Eating Disorder Examination Questionnaire (EDE-Q; Berg, Peterson, Frazier, \& Crow, 2012). WCS scores range from 0 to 100 and were categorized in this study as: low/no concerns (<47), moderate concerns (47-59), and high concerns $(\geq 60)$. These cut-offs are based on prior research in college populations (Jacobi, Abascal, \& Taylor, 2004; Taylor et al., 2006). From the EDE-Q, binge eating in the past 4 weeks was categorized as: no binge eating, 1-3 times, and $\geq 4$ times. Purging was defined as making "yourself sick (vomit) as a means of controlling your shape or weight," taking "laxatives as a means of controlling your shape or weight," taking "diuretics (water pills) or diet pills as a means of controlling your shape or weight," and/or exercising "in a 'driven' or 'compulsive' way as a means of controlling your weight, shape or amount of fat, or to burn off calories" in the past 4 weeks, and was categorized as: no purging, 1-3 times, and $\geq 4$ times. Together, the eating disorder measures capture a continuum of severity and symptom presentation.

\subsection{2 | Suicidality}

In both Study A and Study B, we examined two binary measures of suicidality from the standard HMS mental health status module: (a) suicidal ideation ("In the past year, did you ever seriously think about attempting suicide?"); and (b) suicide attempt ("In the past year, did you attempt suicide?"). Response options were "yes" and "no," and students were categorized accordingly. HMS measures of suicidality were taken from the National Comorbidity Survey (Kessler, Berglund, Borges, Nock, \& Wang, 2005).

\section{4 | Statistical analysis}

Analyses were descriptive in nature, using the cross-sectional HMS survey data. We present results organized by study. In Study A, we estimated the prevalence of suicidal ideation and suicide attempts (weighted percentages) by eating disorder symptom count (number of "yes" answers on the SCOFF, ranging from 0 to 5). We then estimated unadjusted odds ratios (OR) using logistic regressions to examine relationships between suicidality and two operationalizations from the SCOFF: (a) symptom count (reference category $=0$ ); and (b) each of the five items (yes/no). The large, diverse sample in Study A also provided a unique opportunity to assess whether certain individual characteristics captured in the demographics module (age, degree level, race/ethnicity, gender identity, sexual orientation) were associated with increased likelihood of having co-occurring suicidality and eating disorder symptoms. To do so, we estimated ORs for: (a) suicidal ideation with a positive screen for an eating disorder, and (b) suicide attempts with a positive screen for an eating disorder (SCOFF $\geq 2$ ). Reference groups were: 18-22 years (for age), graduate student (for degree level), White (for race/ethnicity), male (for gender identity), and heterosexual (for sexual orientation).

In Study B, we estimated ORs from unadjusted logistic regressions for suicidal ideation and suicide attempts with eating disorder symptoms (WCS, binge eating, purging) as the independent variable of interest. For suicidal ideation, we also estimated adjusted models controlling for the following covariates: (a) age (18-22, 23-25, 26-30, 31 + years); (b) race/ethnicity (White, black, Asian, Latinx, multiracial, other race/ethnicity); (c) gender identity (female, male, transgender, or other gender identity); (d) sexual orientation (heterosexual, bisexual, gay/lesbian, other sexual orientation); (e) weight status (body mass index, based on self-reported height/weight, in three standard categories: underweight $[<18.5]$, healthy weight [18.5-24.9], overweight/ 
TABLE 1 Sample characteristics

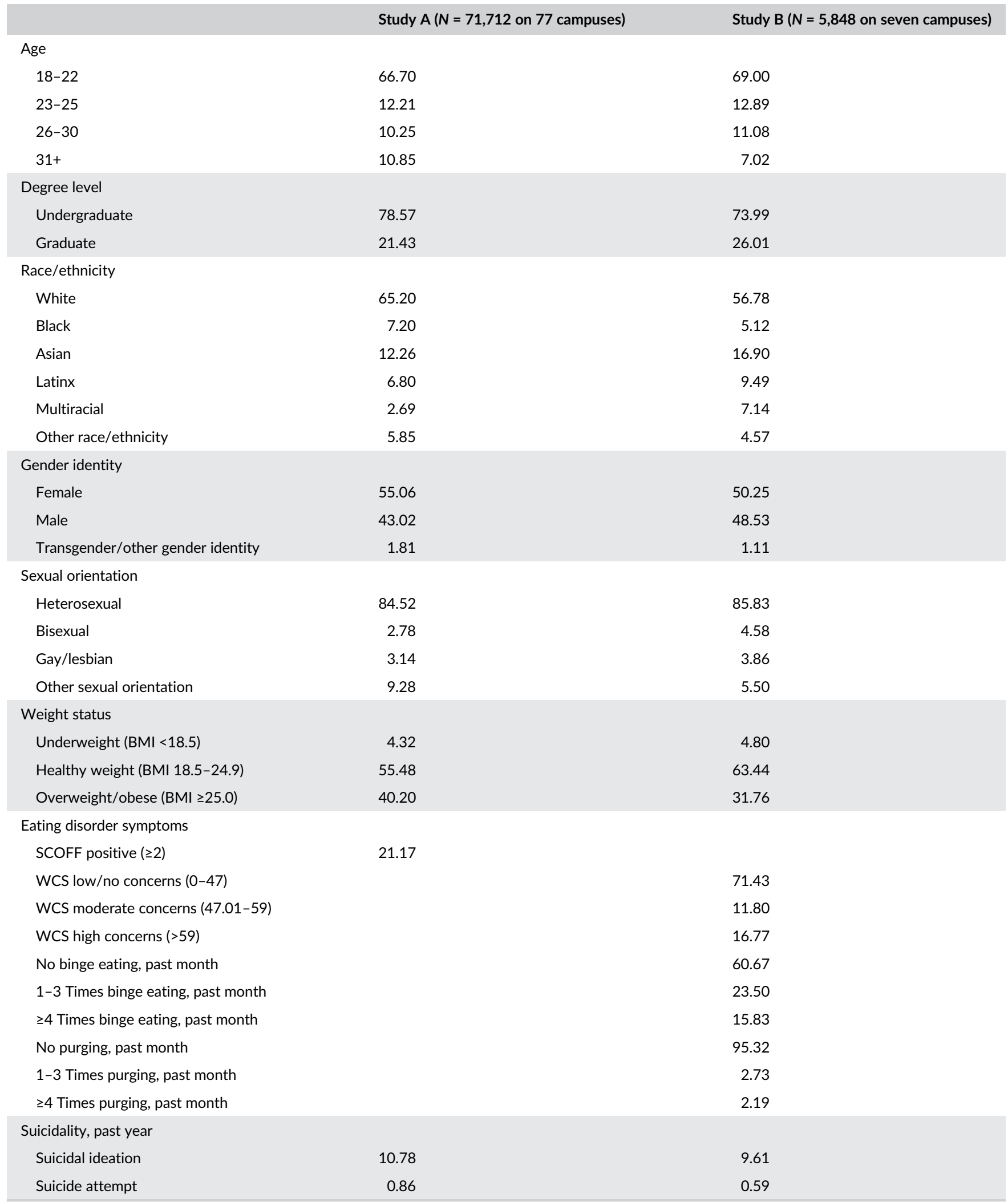

Note: Table values are weighted percentages.

Abbreviations: BMI is body mass index; WCS is Weight Concerns Scale. 
obese [ $\geq 25]$ ); ( $f$ ) symptoms of depression; and (g) symptoms of anxiety. The age categories examined are consistent with extant research using HMS data (Lipson, Kern, Eisenberg, \& Breland-Noble, 2018). Self-reported height and weight were measured in two separate, stand-alone items that were not part of any other scale or screen. Symptoms of depression were measured using the patient health questionnaire-9 (PHQ-9; Kroenke, Spitzer, \& Williams, 2001), operationalized as a binary measure based on the standard cut-off for a positive screen $(\geq 10)$. The PHQ-9 has been validated as internally consistent and highly correlated with diagnosis (Kroenke et al., 2001; Lowe, Unutzer, Callahan, Perkins, \& Kroenke, 2004). Symptoms of anxiety were measured by the generalized anxiety disorder sevenitem (GAD-7) scale, using the cut-off of $\geq 10$, which has been shown to have high sensitivity and specificity (Spitzer, Kroenke, Williams, \& Lowe, 2006). Due to sample size, we were unable to conduct adjusted analyses for suicide attempt. Models were estimated separately for each of the three eating disorder measures (WCS, binge eating, purging), resulting in six models for suicidal ideation and three models for suicide attempts. Analyses were conducted using Stata 15 and weighted using the weights described above.

\section{3 | RESULTS}

\section{1 | Study A}

In Study A, the sample was 71,712 students at 77 U.S. colleges and universities. Just over two-thirds of students were between the ages of 18 and 22, and roughly three-quarters were undergraduates. In terms of race/ethnicity, $65 \%$ identified as White, $7 \%$ as black, $12 \%$ as
Asian, and $7 \%$ as Latinx. There was roughly equal representation of females and males, with 55\% identifying as female. One-in-five students (21\%) screened positive for an eating disorder (SCOFF $\geq 2$ ), $11 \%$ reported past-year suicidal ideation, and $<1 \%$ of the full sample reported attempting suicide. Additional sample characteristics are listed in Table 1.

As presented in Table 2, each additional point on the SCOFF was associated with higher prevalence of past-year suicidality: among students with no symptoms (SCOFF $=0$ ), 7\% reported seriously thinking about attempting suicide relative to $28 \%$ of students with the highest number of symptoms (SCOFF $=5$ ). Even among students with just one symptom of an eating disorder (SCOFF = 1), the prevalence of suicidal ideation was $70 \%$ higher than among those with no symptoms $(12 \%$ versus $7 \%$ ). Similarly, $<1 \%$ of students with no symptoms reported past-year suicide attempts compared to $5 \%$ of those with a score of 5 .

In unadjusted logistic regressions, odds of suicidal ideation and suicide attempt also increased with each additional point on the SCOFF. Relative to a score of 0 , having a score of 1 was associated with two times higher odds of suicidal ideation and suicide attempt, while a score of 2 was associated with three times higher odds of ideation and four times higher odds of attempt $(p<.001)$. Having the highest symptom level was associated with five times higher odds of ideation and 11 times higher odds of attempting suicide $(p<.001)$.

We also examined the five specific symptoms measured in the SCOFF, finding that items \#3 ("Have you recently lost more than 15 pounds in a three-month period?") and \#4 ("Do you believe yourself to be fat when others say you are too thin?") were most strongly correlated with past-year suicidality. Among students who answered "yes" to either of these items, nearly $20 \%$ reported suicidal ideation,

TA B LE 2 Past-year suicidality by eating disorder symptoms, Study A

\begin{tabular}{|c|c|c|c|c|c|c|}
\hline \multirow[b]{2}{*}{ SCOFF symptom count } & \multicolumn{3}{|c|}{ Suicidal ideation } & \multicolumn{3}{|c|}{ Suicide attempt } \\
\hline & $\%$ & OR (SE) & $95 \% \mathrm{Cl}$ & $\%$ & OR (SE) & $95 \% \mathrm{Cl}$ \\
\hline 0 & 6.95 & REF & & 0.46 & REF & \\
\hline 1 & 12.09 & $1.84(0.08) * * *$ & $1.70,2.00$ & 0.77 & $1.69(0.26) * *$ & $1.24,2.29$ \\
\hline 2 & 17.30 & $2.80(0.13) * * *$ & $2.56,3.06$ & 1.58 & $3.47(0.53) * * *$ & $2.56,4.68$ \\
\hline 3 & 20.71 & $3.50(0.19) * * *$ & $3.15,3.89$ & 2.14 & $4.72(0.80) * * *$ & $3.39,6.59$ \\
\hline 4 & 27.54 & $5.09(0.41) * * *$ & $4.35,5.96$ & 3.74 & $8.40(1.77) * * *$ & $5.55,12.72$ \\
\hline 5 & 28.00 & $5.21(0.85) * * *$ & $3.79,7.16$ & 4.84 & $11.02(4.43) * * *$ & $5.01,24.23$ \\
\hline \multicolumn{7}{|l|}{ SCOFF items } \\
\hline Item \#1 (make yourself sick) & 18.26 & $2.09(0.08) * * *$ & $1.94,2.25$ & 1.97 & $2.89(0.34) * * *$ & $2.29,3.64$ \\
\hline $\begin{array}{l}\text { Item \#2 (worry you have lost control over how much you } \\
\text { eat) }\end{array}$ & 17.21 & $2.27(0.07) * * *$ & $2.13,2.42$ & 1.50 & $2.44(0.26) * * *$ & $1.97,3.01$ \\
\hline Item \#3 (recently lost more than 15 pounds) & 19.88 & $2.24(0.18) * * *$ & $2.04,2.46$ & 2.21 & $3.03(0.39) * * *$ & $2.35,3.91$ \\
\hline $\begin{array}{l}\text { Item \#4 (believe to be fat when others say you are too } \\
\text { thin) }\end{array}$ & 19.22 & $2.29(0.09) * * *$ & $2.12,2.46$ & 2.11 & $3.26(0.36) * * *$ & $2.62,4.05$ \\
\hline Item \#5 (food dominates your life) & 16.28 & $1.78(0.07) * * *$ & $1.64,1.92$ & 1.56 & $2.11(0.25) * * *$ & $1.68,2.65$ \\
\hline
\end{tabular}

Note: Table values are weighted percentages and odds ratios (OR) from unadjusted logistic regressions with standard errors (SE) in parentheses. $95 \%$ Confidence intervals are presented in the far right column for each outcome. For SCOFF symptom count, the reference category (REF) was 0.

$* * * p<.001 ; * * p<.01$ 
and a "yes" answer to either of these items was associated with three times higher odds of suicide attempt $(p<.001)$.

As presented in Table 3, $4 \%$ of the sample $(N=2,912)$ reported both suicidal ideation and screened positive for an eating disorder, while $0.4 \%(N=288)$ reported making a suicide attempt and screened positive for an eating disorder. The following characteristics were associated with significantly higher odds of having co-occurring eating disorder symptoms and suicidality (both suicidal ideation and suicide attempt), all at $p<.001$ : undergraduate (relative to graduate student); female and transgender/other gender identity (relative to male); and bisexual, gay/lesbian, and other sexual orientation (relative to heterosexual). Relative to being ages 18-22, all other age groups had significantly lower odds for co-occurring eating disorder symptoms and suicidal ideation; this pattern remained the same for suicide attempt, though only age 31+ was statistically significant at the level of $p<.001$. Having overweight/obesity (relative to healthy weight) was also associated with significantly higher odds of co-occurring suicidal ideation and eating disorder symptoms.

\section{2 | Study B}

In Study B, the sample was 5,848 students at seven colleges and universities that elected the eating disorder module. Roughly two-thirds of students were between ages 18-22 and three-quarters were undergraduates. Just over half identified as White, and the sample was $50 \%$ female. On the WCS, overall $71 \%$ were classified as having low/no weight and shape concerns (63\% for females, $81 \%$ for males), $12 \%$ as having moderate concerns, and $17 \%$ as having high concerns.

TAB LE 3 Cooccurring suicidality and eating disorder symptoms, variations by individual characteristics, Study A

\begin{tabular}{|c|c|c|c|c|}
\hline \multirow{2}{*}{$\begin{array}{l}\text { Age } \\
18-22\end{array}$} & \multicolumn{2}{|c|}{ Suicidal ideation and SCOFF >2 } & \multicolumn{2}{|c|}{ Suicide attempt and SCOFF $>2$} \\
\hline & REF & & REF & \\
\hline $23-25$ & $0.70(0.06) * * *$ & $0.60,0.83$ & $0.68(0.17)$ & $0.41,1.13$ \\
\hline $26-30$ & $0.58(0.05) * * *$ & $0.48,0.69$ & $0.50(0.13) * *$ & $0.29,0.84$ \\
\hline $31+$ & $0.44(0.04) * * *$ & $0.36,0.54$ & $0.25(0.09) * * *$ & $0.12,0.50$ \\
\hline \multicolumn{5}{|l|}{ Degree level } \\
\hline Undergraduate & $1.99(0.14) * * *$ & $1.73,2.29$ & $2.87(0.67) * * *$ & $1.82,4.55$ \\
\hline Graduate & REF & & REF & \\
\hline \multicolumn{5}{|l|}{ Race/ethnicity } \\
\hline White & REF & & REF & \\
\hline Black & $0.80(0.10)$ & $0.62,1.03$ & $1.00(0.30)$ & $0.56,1.80$ \\
\hline Asian & $0.97(0.07)$ & $0.84,1.13$ & $1.51(0.31) *$ & $1.02,2.25$ \\
\hline Latinx & $1.18(0.11)$ & $0.99,1.40$ & $1.23(0.34)$ & $0.72,2.10$ \\
\hline Multiracial & $1.51(0.18) * *$ & $1.19,1.91$ & $2.38(0.86) *$ & $1.17,4.84$ \\
\hline Other race/ethnicity & $1.30(0.13)^{* *}$ & $1.08,1.58$ & $1.56(0.43)$ & $0.90,2.69$ \\
\hline \multicolumn{5}{|l|}{ Gender identity } \\
\hline Female & $1.90(0.12)^{* * *}$ & $1.69,2.14$ & $2.39(0.45) * * *$ & $1.65,3.47$ \\
\hline Male & REF & & REF & \\
\hline Transgender/other gender identity & $7.37(0.83) * * *$ & $5.91,9.19$ & $8.25(2.70) * * *$ & $4.34,15.69$ \\
\hline \multicolumn{5}{|l|}{ Sexual orientation } \\
\hline Heterosexual & REF & & REF & \\
\hline Bisexual & $3.61(0.34) * * *$ & $3.01,4.34$ & $5.20(1.57) * * *$ & $2.88,9.39$ \\
\hline Gay/lesbian & $2.56(0.30) * * *$ & $2.04,3.20$ & $4.05(1.08) * * *$ & $2.40,6.85$ \\
\hline Other sexual orientation & $4.19(0.24) * * *$ & $3.74,4.68$ & $5.56(0.91) * * *$ & $4.02,7.67$ \\
\hline \multicolumn{5}{|l|}{ Weight status } \\
\hline Underweight (BMI <18.5) & $0.98(0.13)$ & $0.77,1.27$ & $1.75(0.61)$ & $0.88,3.45$ \\
\hline Healthy weight (BMI 18.5-24.9) & REF & & REF & \\
\hline Overweight/obese (BMI $\geq 25.0$ ) & $1.48(0.07) * * *$ & $1.34,1.62$ & $1.50(0.22) * *$ & $1.12,2.01$ \\
\hline
\end{tabular}

Note: Table values are odds ratios (OR) from unadjusted logistic regressions with standard errors (SE) in parentheses. 95\% Confidence intervals are presented in the far right column for each outcome. The dependent variables were: (a) suicidal ideation with a positive screen for an eating disorder (SCOFF > 2); and (b) suicide attempt with a positive screen for an eating disorder (SCOFF $\geq 2$ ). Reference groups (REF) were: 18-22 years (for age), graduate student (for degree level), white (for race/ethnicity), male (for gender identity), heterosexual (for sexual orientation), and healthy weight (for weight status).

Abbreviation: BMI, body mass index.

$* * * p<.001 ; * * p<.01 ; * p<.05$. 
TAB LE 4 Unadjusted and adjusted correlates of past-year suicidality by eating disorder symptoms, Study B

\begin{tabular}{|c|c|c|c|c|c|c|}
\hline & \multicolumn{4}{|c|}{ Suicidal ideation } & \multirow{2}{*}{\multicolumn{2}{|c|}{$\begin{array}{l}\text { Suicide attempt } \\
\text { Unadjusted }\end{array}$}} \\
\hline & Unadjusted & & Adjusted & & & \\
\hline WCS low/no concerns & REF & & REF & & REF & \\
\hline WCS moderate concerns & $1.54(0.24) * *$ & $1.14,2.09$ & $1.36(0.23)$ & $0.98,1.90$ & $1.61(0.88)$ & $0.55,4.71$ \\
\hline WCS high concerns & $2.12(0.25) * * *$ & $1.68,2.68$ & $1.46(0.21) * *$ & $1.09,1.93$ & $3.01(1.35) *$ & $1.25,7.25$ \\
\hline No binge eating, past month & REF & & REF & & REF & \\
\hline 1-3 Times binge eating, past month & $1.11(0.14)$ & $0.86,1.43$ & $0.89(0.13)$ & $0.67,1.17$ & $1.14(0.61)$ & $0.40,3.27$ \\
\hline$\geq 4$ Times binge eating, past month & $2.15(0.27) * * *$ & $1.69,2.75$ & $1.16(0.17)$ & $0.87,1.54$ & $2.24(1.00)$ & $0.94,5.37$ \\
\hline No purging, past month & REF & & REF & & REF & \\
\hline 1-3 Times purging, past month & $1.93(0.49) * *$ & $1.18,3.17$ & $1.39(0.38)$ & $0.82,2.36$ & $4.12(2.39) *$ & $1.32,12.86$ \\
\hline$\geq 4$ Times purging, past month & $2.81(0.74) * * *$ & $1.67,4.71$ & $1.65(0.49)$ & $0.92,2.96$ & $7.28(4.82) * *$ & $1.99,26.66$ \\
\hline
\end{tabular}

Note: Table values are odds ratios with standard errors in parentheses. $95 \%$ Confidence intervals are presented in the far right column for each outcome (for both unadjusted and adjusted models for suicidal ideation). All models were estimated separately for each of the three eating disorder measures (WCS, binge eating, and purging), resulting in six models for suicidal ideation and three models for suicide attempts. Due to sample size, we were unable to conduct adjusted analyses for suicide attempt. The adjusted logistic regression models for past-year suicidal ideation controlled for age, race/ethnicity, gender identity, sexual orientation, weight status, depression (PHQ-9 $\geq 10$ ), and anxiety (GAD-7 $\geq 10$ ). Reference categories (REF) were: WCS low/no concerns; no binge eating; and no purging. Abbreviations: GAD-7, generalized anxiety disorder scale-7; PHQ-9, patient health questionnaire-9; WCS, weight concerns scale. $* * * p<.001 ; * *<.01 ; * p<.05$.

The mean WCS score was 41 for females, 29 for males, and 35 for transgender students/students with another gender identity. For binging, $61 \%$ reported no binge eating in the past month, $24 \%$ reported one to three times, and $16 \%$ reported $\geq 4$ times. For purging, $95 \%$ reported no purging, $3 \%$ reported one to three times, and $2 \%$ reported $\geq 4$ times. One-in-ten students reported suicidal ideation and $<1 \%$ reported a suicide attempt. Additional sample characteristics are presented in Table 1.

In unadjusted logistic regressions, moderate weight and shape concerns were associated with 1.5 times higher odds of suicidal ideation ( $p=.005)$ and high concerns with 2.1 times higher odds $(p<.001$; Table 4). Relative to no binge eating, $\geq 4$ times binge eating was associated with 2.2 times higher odds of suicidal ideation $(p<.001)$. Relative to no purging, purging one to three times was associated with 1.9 times higher odds $(p=.009)$ and purging $\geq 4$ times with 2.8 times higher odds ( $p<.001$ ) of suicidal ideation. In the adjusted models controlling for demographic characteristics and symptoms of depression and anxiety, high weight, and shape concerns were associated with significantly increased odds of suicidal ideation. Other tested associations were no longer significant in the adjusted models once controlling for demographics, depression, and anxiety.

In the unadjusted models for past-year suicide attempt, high weight and shape concerns were associated with 3.0 times higher odds ( $p=.014$ ), while purging one to three times was associated with 4.1 times higher odds ( $p=.015$ ), and purging $\geq 4$ times with 7.3 times higher odds $(p=.003)$.

\section{4 | DISCUSSION}

Eating disorders have the highest rate of mortality of any mental illness (Pisetsky et al., 2013), and suicide is a leading cause of death for individuals with eating disorders (Arcelus et al., 2011; Sullivan, 1995). Despite a national dialogue about suicide on college campuses, little is known about the relationship between eating disorder symptoms and suicide risk. Echoing national trends in the high and rising prevalence of mental health problems among college students (Eisenberg et al., 2007; Eisenberg, Hunt, \& Speer, 2013; Eisenberg, Nicklett, Roeder, \& Kirz, 2011; Fernández Rodríguez \& Huertas, 2013; Kraft, 2011; Lipson, Heinze, Gaddis, Beck, \& Eisenberg, 2015; Schwartz, 2006), this study underscores the population-level burden of both suicidality and eating disorder symptoms, with one-in-five students in our sample meeting criteria for indicated eating disorder risk and roughly one-inten reporting having seriously considered attempting suicide in the past year.

From a public health perspective, little is known about the relationship between suicide risk and symptoms of eating disorders, in the college setting or otherwise. This population-level study of a large, random sample of students is an important compliment to previous research, which has focused primarily on clinical samples or specific subsets of students, such as female athletes or sorority women (Allison \& Park, 2004; Harris et al., 2015; Wollenberg et al., 2015). This study drew from a large sample of college students on campuses across the United States to assess risk of suicidality along a continuum of eating disorder symptomatology, examining symptom severity, weight and shape concerns, and specific behaviors of binging and purging.

Consistent with extant research (Ackard et al., 2003; Crow et al., 2008a; Crow et al., 2008b; Pompili, Girardi, Tatarelli, Ruberto, \& Tatarelli, 2006; Preti, Rocchi, Sisti, Camboni, \& Miotto, 2011; Rafiroiu et al., 2003; Rodríguez-Cano et al., 2006), we saw a significant association between eating disorder symptoms and suicidality. In Study A, the prevalence of suicidal ideation and attempts increased with each 
additional symptom count on the SCOFF. Our findings suggest that endorsement of any symptoms of an eating disorder (i.e., SCOFF = 1) could signal elevated risk of suicidality. Recent weight loss and body dysmorphia (believing "yourself to be fat when others say you are too thin") were the most predictive symptoms of suicidal ideation and suicide attempts. In this diverse sample drawn from 77 campuses, we found that younger students, undergraduates, females, transgender students, queer students (bisexual, gay, lesbian, other sexuality), and students with overweight/obesity were at increased risk for having co-occurring eating disorders and suicidality.

In Study B, we found that weight and shape concerns were associated with higher rates of suicidality, even after controlling for individual characteristics and adjusting for psychiatric comorbidity. Consistent with other studies (Favaro \& Santonastaso, 1997; Franko \& Keel, 2006; Goel et al., 2018; Pisetsky et al., 2013), we observed a strong association between suicide attempts and eating disorder presentations that include purging behaviors.

\section{1 | Limitations}

As noted, this is the largest known study to date examining associations between suicidality and eating disorder symptoms in college populations. Generalizability is strengthened by the multisite nature of our data and the use of a random sampling approach at the student-level. Though college students are an important population for this research due to epidemiological vulnerability, the findings may not be generalizable to same-aged non-college-attending peers, who tend to differ across numerous demographic and social dimensions. The study also provided a rare opportunity to examine eating disorder symptomatology using three validated measures (SCOFF, WCS, and EDE-Q binge-purge items). In addition to these strengths, there are several limitations to consider when interpreting results. First, campuses elected to participate in HMS and may not be representative of other institutions. In particular, campuses that selected the eating disorder elective module (Study B) may have done so out of a recognized need to address this particular issue in their student body. Though the SCOFF measure was analyzed only in Study A, these five questions were also asked of students in Study B. To address this potential limitation, we estimated separately the prevalence of positive SCOFF screens among students on the 70 campuses that did not elect the eating disorder module and compared this with the seven campuses that elected that module. The prevalence of positive screens was actually slightly higher on the 70 campuses that did not administer the elective module (21.4\%) than on the seven campuses that did (18.8\%), though this difference was not statistically significant. Third, the survey response rates was $27 \%$ in $2015-2016$ and $23 \%$ in $2016-2017$. While this is typical for an online survey of this nature (Eisenberg et al., 2007), it raises concerns of response bias. As described, we applied sample probability weights to address concerns related to systematic differences between respondents and nonrespondents along known characteristics of the full population, but there may be differences on unobserved characteristics, including eating disorder symptoms and suicidality. Finally, we were unable to examine temporal relationships due to the cross-sectional nature of the data. Future longitudinal studies are needed to explore the temporality of eating disorders and suicidality.

\section{5 | CONCLUSION}

Findings from this study have important implications for prevention and early intervention, particularly in terms of screening and identification of both suicide risk and eating disorders. Given rising rates of student suicides, many colleges and universities are deeply concerned with suicide prevention (Schwartz, 2006). Findings from this study suggest that screening for eating disorders should be a priority within broader suicide prevention efforts. Rather than assessing a single aspect of student mental health (e.g., depression), as is common in suicide prevention efforts on college campuses (Eisenberg \& Lipson, 2019; Fernández Rodríguez \& Huertas, 2013) comprehensive mental health screenings are needed in order to collectively assess risk for suicide, eating disorders, and other prevalent mental health conditions. In our sample, younger students were at increased risk for cooccurring suicide and eating disorders. There is an urgent need and unique opportunity for screening efforts during the first undergraduate year, a time marked simultaneously by significant psychosocial transition and epidemiological vulnerability for eating disorders coupled with high and rising rates of suicidality (Schwartz, 2006).

Furthermore, this study points to a need for assessing eating disorders along a continuum of severity and symptom presentation. Eating disorder symptoms, even at what would be considered subthreshold, were highly predictive of suicide risk in this national sample. Screening, prevention, and intervention efforts should recognize the particular relationship between suicide and purging behaviors, both of which have been linked to impulsivity (Claes, Nederkoorn, Vandereycken, Guerrieri, \& Vertommen, 2006).

Findings from this study also contribute to a growing understanding of mental health disparities in college populations. This study provides the first known evidence of how eating disorder and suicide risk varies across student characteristics. In our sample, students from traditionally underrepresented, marginalized backgrounds had higher rates of co-occurring eating disorders and suicide risk. Targeted outreach and tailored prevention and intervention approaches are needed for vulnerable students on campus, including sexual and gender minority students who were between four and eight times more likely to have a positive eating disorder screen coupled with attempted suicide. Though it is still not well recognized, students with overweight and obesity are at increased risk not only for eating disorders and suicide, but are also less likely to be identified and treated due to weight bias in health systems (Lipson \& Sonneville, 2017).

Overall, results from this study add to a growing literature that underscores the critical need for the application of population-level approaches to suicide and eating disorder prevention (Austin, 2012), and provides support for integration of mental health prevention and early intervention efforts on college campuses. 


\section{DATA AVAILABILITY STATEMENT}

The data that support the findings of this study are available upon request from the Healthy Minds research team. Please visit www. healthymindsnetwork.org/research/data-for-researchers for more information

\section{REFERENCES}

Ackard, D., Neumark-Sztainer, D., Story, M., \& Perry, C. (2003). Overeating among adolescents: Prevalence and associations with weight-related characteristics and psychological health. Pediatrics, 111(1), 67-74.

Allison, K., \& Park, C. (2004). A prospective study of disordered eating among sorority and nonsorority women. International Journal of Eating Disorders, 35(3), 354-358. https://doi.org/10.1002/eat.10255

Arcelus, J., Mitchell, A., Wales, J., \& Nielsen, S. (2011). Mortality rates in patients with anorexia nervosa and other eating disorders a metaanalysis of 36 studies. Archives of General Psychiatry, 68(7), 724-731.

Austin, S. (2012). A public health approach to eating disorders prevention: It's time for public health professionals to take a seat at the table. BMC Public Health, 12(1), 854-856. https://doi.org/10.1186/1471-245812-854

Berg, K., Peterson, C., Frazier, P., \& Crow, S. (2012). Psychometric evaluation of the eating disorder examination and eating disorder examination-questionnaire: A systematic review of the literature. International Journal of Eating Disorders, 45(3), 428-438. https://doi.org/ 10.1002/eat.20931

Bodell, L., Joiner, T., \& Keel, P. (2013). Comorbidity-independent risk for suicidality increases with bulimia nervosa but not with anorexia nervosa. Journal of Psychiatric Research, 47(5), 617-621. https://doi. org/10.1016/j.jpsychires.2013.01.005

Claes, L., Nederkoorn, C., Vandereycken, W., Guerrieri, R., \& Vertommen, H. (2006). Impulsiveness and lack of inhibitory control in eating disorders. Eating Behaviors, 7(3), 196-203. https://doi.org/10. 1016/j.eatbeh.2006.05.001

Conti, C., Lanzara, R., Scipioni, M., lasenza, M., Guagnano, M., \& Fulcheri, M. (2017). The relationship between binge eating disorder and suicidality: A systematic review. Frontiers in Psychology, 8, 2125. https://doi.org/10.3389/fpsyg.2017.02125

Crow, S., Eisenberg, M., Story, M., \& Neumark-Sztainer, D. (2008a). Suicidal behavior in adolescents: Relationship to weight status, weight control behaviors, and body dissatisfaction. International Journal of Eating Disorders, 41(1), 82-87. https://doi.org/10.1002/eat.20466

Crow, S., Eisenberg, M., Story, M., \& Neumark-Sztainer, D. (2008b). Are body dissatisfaction, eating disturbance, and body mass index predictors of suicidal behavior in adolescents? A longitudinal study. Journal of Consulting and Clinical Psychology, 76(5), 887-892. https://doi.org/ 10.1037/a0012783

Eichen, D., Kass, A., Fitzsimmons-Craft, E., Gibbs, E., Trockel, M., Taylor, C. B., \& Wilfley, D. (2016). Non-suicidal self-injury and suicidal ideation in relation to eating and general psychopathology among college-age women. Psychiatry Research, 235, 77-82.

Eisenberg, D., Golberstein, E., \& Gollust, S. (2007). Help-seeking and access to mental health Care in a University Student Population. Medical Care, 45(7), 594-601.

Eisenberg, D., Hunt, J. B., \& Speer, N. (2013). Mental health in American colleges and universi- ties: Variation across student subgroups and across campuses. Journal of Nervous and Mental Disease, 201(1), 60-67. https://doi.org/10.1097/nmd.0b013e31827ab077

Eisenberg, D., \& Lipson, SK. (2019). The Healthy Minds Study. Retrieved from: www.healthymindsnetwork.org/hms.

Eisenberg, D., Nicklett, E., Roeder, K., \& Kirz, N. (2011). Eating disorder symptoms among college students: Prevalence, persistence, correlates, and treatment-seeking. Journal of American College Health, 59(8), 700-707.

Favaro, A., \& Santonastaso, P. (1997). Suicidality in eating disorders: Clinical and psychological correlates. Acta Psychiatrica Scandinavica, 95(6), 508-514. https://doi.org/10.1111/j.1600-0447.1997.tb10139.x

Fernández Rodríguez, M., \& Huertas, I. (2013). Suicide prevention in college students: A collaborative approach. Interamerican Journal of Psychology, 47(1), 53-60.

Fichter, M., \& Quadflieg, N. (2016). Mortality in eating disorders - results of a large prospective clinical longitudinal study. International Journal of Eating Disorders, 49(4), 391-401. https://doi.org/10.1002/eat. 22501

Forrest, L., Zuromski, K., Dodd, D., \& Smith, A. (2017). Suicidality in adolescents and adults with binge-eating disorder: Results from the national comorbidity survey replication and adolescent supplement. International Journal of Eating Disorders, 50(1), 40-49. https://doi.org/10. 1002/eat.22582

Franko, D., \& Keel, P. (2006). Suicidality in eating disorders: Occurrence, correlates, and clinical implications. Clinical Psychology Review, 26(6), 769-782. https://doi.org/10.1016/j.cpr.2006.04.001

Goel, N., Sadeh-Sharvit, S., Flatt, R., Trockel, M., Balantekin, K., Fitzsimmons-Craft, E., ... Taylor, C. B. (2018). Correlates of suicidal ideation in college women with eating disorders. International Journal of Eating Disorders, 51(6), 579-584.

Harris, N., Gee, D., D'Acquisto, D., Ogan, D., \& Pritchett, K. (2015). Eating disorder risk, exercise dependence, and body weight dissatisfaction among female nutrition and exercise science university majors. Journal of Behavioral Addictions, 4(3), 206-209. https://doi.org/10.1556/ 2006.4.2015.029

Herzog, D., Greenwood, D., Dorer, D., Flores, A., Ekeblad, E., Richards, A., ... Keller, M. (2000). Mortality in eating disorders: A descriptive study. International Journal of Eating Disorders, 28(1), 20-26. https://doi.org/ 10.1002/(SICI)1098-108X(200007)28:1<20::AID-EAT3>3.0.CO2-X

Jacobi, C., Abascal, L., \& Taylor, C. (2004). Screening for eating disorders and high-risk behavior: Caution. International Journal of Eating Disorders, 36(3), 280-295. https://doi.org/10.1002/eat.20048

Kessler, R., Berglund, P., Borges, G., Nock, M., \& Wang, P. (2005). Trends in suicide ideation, plans, gestures, and attempts in the United States, 1990-1992 to 2001-2003. Jama, 293(20), 2487-2495.

Killen, J., Taylor, C., Hayward, C., Wilson, D., Haydel, K., Hammer, L., ... Kraemer, H. (1994). Pursuit of thinness and onset of eating disorder symptoms in a community sample of adolescent girls: A three-year prospective analysis. International Journal of Eating Disorders, 16(3), 227-238. https://doi.org/10.1002/1098-108X(199411)16:3<227:: AID-EAT2260160303>3.0.CO2-L

Kraft, D. P. (2011). One hundred years of college mental health. Journal of American College Health, 59(6), 477-481. https://doi.org/10.1080/ 07448481.2011 .569964

Kroenke, K., Spitzer, R., \& Williams, J. (2001). The PHQ-9. Journal of General Internal Medicine, 16(9), 606-613. https://doi.org/10.1046/j. 1525-1497.2001.016009606.x

Lipson, S., \& Sonneville, K. (2017). Eating disorder symptoms among undergraduate and graduate students at $12 \mathrm{U}$. S. Colleges and universities. Eating Behaviors, 24, 81-88. https://doi.org/10.1016/j.eatbeh. 2016.12.003

Lipson, S. K., Heinze, J., Gaddis, S. M., Beck, K., \& Eisenberg, D. (2015). Variations in student mental health and treatment utilization across U. S. Colleges and universities. Journal of American College Health, 63 (6), 388-396. https://doi.org/10.1080/07448481.2015.1040411

Lipson, S., Kern, A., Eisenberg, D., \& Breland-Noble, A. (2018). Mental health disparities among college students of color. Journal of Adolescent Health, 63, 348-356.

Lowe, B., Unutzer, J., Callahan, C. M., Perkins, A. J., \& Kroenke, K. (2004). Monitoring depression treatment outcomes with the patient health questionnaire-9. Medical Care, 42(12), 1194-1201. 
Luck, A., Morgan, J., Reid, F., O'Brien, A., Brunton, J., Price, C., ... Lacey, J. (2002). The SCOFF questionnaire and clinical interview for eating disorders in general practice: Comparative study. BMJ: British Medical Journal, 325(7367), 755-756.

Maguen, S., Hebenstreit, C., Li, Y., Dinh, J., Donalson, R., Dalton, S., ... Masheb, R. (2018). Screen for disordered eating: Improving the accuracy of eating disorder screening in primary care. General Hospital Psychiatry, 50, 20-25.

Morgan, J., Reid, F., \& Lacey, J. (1999). The SCOFF questionnaire: Assessment of a new screening tool for eating disorders. BMJ: British Medical Journal, 319(7223), 1467-1468.

Pisetsky, E., Thornton, L., Lichtenstein, P., Pedersen, N., \& Bulik, C. (2013). Suicide attempts in women with eating disorders. Journal of Abnormal Psychology, 122(4), 1042-1056. https://doi.org/10.1037/a0034902

Pompili, M., Girardi, P., Tatarelli, G., Ruberto, A., \& Tatarelli, R. (2006). Suicide and attempted suicide in eating disorders, obesity and weightimage concern. Eating Behaviors, 7(4), 384-394. https://doi.org/10. 1016/j.eatbeh.2005.12.004

Preti, A., Rocchi, M., Sisti, D., Camboni, M., \& Miotto, P. (2011). A comprehensive meta-analysis of the risk of suicide in eating disorders. Acta Psychiatrica Scandinavica, 124(1), 6-17. https://doi.org/10.1111/j. 1600-0447.2010.01641.x

Rafiroiu, A., Sargent, R., Parra-Medina, D., Drane, W., \& Valois, R. (2003). Covariations of adolescent weight-control, health-risk and healthpromoting behaviors. American Journal of Health Behavior, 27(1), 3-14.

Rodríguez-Cano, T., Beato-Fernández, L., \& Llario, A. (2006). Body dissatisfaction as a predictor of self-reported suicide attempts in adolescents: A Spanish community prospective study. Journal of Adolescent Health, 38(6), 684-688. https://doi.org/10.1016/j.jadohealth.2005.08.003

Schwartz, A. (2006). College student suicide in the United States: 1990-1991 through 2003-2004. Journal of American College Health, 54(6), 341-352. https://doi.org/10.3200/JACH.54.6.341-352

Sullivan, P. (1995). Mortality in anorexia nervosa. The American Journal of Psychiatry, 152(7), 1073-1074.

Spitzer, R., Kroenke, K., Williams, J., \& Lowe, B. (2006). A brief measure for assessing generalized anxiety disorder: The GAD-7. Archives of Internal
Medicine, 166(1), 1092-1097. https://doi.org/10.1001/archinte.166. 10.1092

Suokas, J., Suvisaari, J., Gissler, M., Löfman, R., Linna, M., Raevuori, A., \& Haukka, J. (2013). Mortality in eating disorders: A follow-up study of adult eating disorder patients treated in tertiary care, 1995-2010. Psychiatry Research, 210(3), 1101-1106. https://doi.org/10.1016/j. psychres.2013.07.042

Taylor, C., Bryson, S., Luce, K., Cunning, D., Doyle, A., Abascal, L., ... Wilfley, D. (2006). Prevention of eating disorders in at-risk college-age women. Archives of General Psychiatry, 63(8), 881-888.

Udo, T., \& Grilo, C. (2018). Prevalence and correlates of DSM-5-defined eating disorders in a nationally representative sample of U. S. Adults. Biological Psychiatry, 84(5), 345-354. https://doi.org/10.1016/j. biopsych.2018.03.014

Wade, T., Fairweather-Schmidt, A., Zhu, G., \& Martin, N. (2015). Does shared genetic risk contribute to the co-occurrence of eating disorders and suicidality? International Journal of Eating Disorders, 48(6), 684-691. https://doi.org/10.1002/eat.22421

Wollenberg, G., Shriver, L., \& Gates, G. (2015). Comparison of disordered eating symptoms and emotion regulation difficulties between female college athletes and non-athletes. Eating Behaviors, 18, 1-6. https:// doi.org/10.1016/j.eatbeh.2015.03.008

Yao, S., Kuja-Halkola, R., Thornton, L., Runfola, C., D'Onofrio, B., Almqvist, C., ... Sjolander, A. (2016). Familial liability for eating disorders and suicide attempts: Evidence from a population registry in Sweden. JAMA Psychiatry, 73(3), 284-291. https://doi.org/10.1001/ jamapsychiatry.2015.2737

How to cite this article: Lipson SK, Sonneville KR. Understanding suicide risk and eating disorders in college student populations: Results from a National Study. Int J Eat Disord. 2020;53:229-238. https://doi.org/10.1002/eat.23188 